\title{
Health-Related Quality of Life Among Children with Acute Lymphoblastic Leukemia
}

\author{
William Furlong, MSc ${ }^{1,2}$, Charlene Rae, MRM, MSc ${ }^{1}$, David Feeny, BA, MA, PhD ${ }^{2,3}$, Richard \\ D Gelber, $\mathrm{PhD}^{4}$, Caroline Laverdiere, $\mathbf{M D}^{5}$, Bruno Michon, $\mathbf{M D}^{6}$, Lewis Silverman, $\mathbf{M D}^{4}$, \\ Stephen Sallan, $\mathbf{M D}^{4}$, and Ronald Barr, MB, ChB, MD ${ }^{7}$ \\ ${ }^{1}$ Centre for Health Economics and Policy Analysis, and Department of Clinical Epidemiology and \\ Biostatistics, McMaster University, Hamilton ON, Canada. \\ 2 Health Utilities Inc., Dundas ON, Canada. \\ 3 The Center for Health Research, Kaiser Permanente Northwest Region, Portland OR, USA. \\ ${ }^{4}$ Dana Farber Cancer Institute, and Children's Hospital, Boston MA, USA. \\ ${ }^{5}$ Hôpital Sainte Justine, Montreal QC, Canada. \\ ${ }^{6}$ Le Centre Hospitalier de L'Universite Laval, Québec City QC, Canada. \\ 7 Department of Pediatrics, McMaster University, Hamilton ON, Canada and McMaster Children's \\ Hospital, Hamilton ON, Canada.
}

\begin{abstract}
BACKGROUND-The objective was to quantify the health-related quality of life (HRQL) of children treated for acute lymphoblastic leukemia (ALL) and identify specific disabilities for remediation.
\end{abstract}

PROCEDURE-Two types of subjects were included: ALL patients 5 plus years old in a multicenter clinical trial; and general population control groups. Patients were assessed during all 4 major phases of active treatment and approximately 2 years after treatment. Health status and HRQL were measured using HEALTH UTILITIES INDEX® (HUI®) Mark 2 (HUI2) and Mark 3 (HUI3). HRQL scores were used to calculate quality-adjusted life years (QALYs). Excess disability rates identified attributes for remediation.

\begin{abstract}
RESULTS-HUI assessments ( $\mathrm{n}=749$ ) were collected during the 5 phases. Mean HRQL increased from induction through the post-treatment phase $(\mathrm{p}<0.001)$. There were no significant demographic or treatment effects on HRQL, except for type of asparaginase during continuation therapy ( $\mathrm{p}=0.005$ for HUI2 and $\mathrm{p}=0.007$ for HUI3). Differences in mean HRQL scores between patients and controls were important $(\mathrm{p}<0.001)$ during the active treatment phases but not during the post-treatment phase. Relative to controls, patients lost approximately 0.2 QALYs during
\end{abstract}

Correspondence to: William Furlong, MSc, CRL 217, McMaster University, 1200 Main Street West, Hamilton ON L8N-3Z5, Canada. Telephone: (905) 525-9140, x22389. Fax: (905) 546-5211. furlongb@mcmaster.ca..

Conflict of Interest Statement. William Furlong and David Feeny have a proprietary interest in, and are officers of, Health Utilities Incorporated (HUInc). HUInc distributes copyrighted HEALTH UTILITIES INDEX® (HUI®) materials and provides methodological advice on the use of HUI. None of the other authors declare any conflict of interest. 
active treatment. Disability was evident in mobility/ambulation, emotion, self-care and pain, and declined over time.

CONCLUSIONS-Patients with ALL experienced important but declining deficits in HRQL during active treatment phases: equivalent to losing approximately 2 months of life in perfect health. HRQL within the 2-years post-treatment phase was similar to controls. The policy challenge is to develop new treatment protocols producing fewer disabilities in mobility/ ambulation, emotion, self-care, and pain without compromising survival.

\section{Keywords}

acute lymphoblastic leukemia; quality of life; outcomes research; disability; survivors; treatment

\section{INTRODUCTION}

Worldwide, thousands of children and adolescents are diagnosed with acute lymphoblastic leukemia (ALL) each year. Approximately 2,300 cases occur annually in the United States. Major advances in treatment have increased survival rates from less than 50\% in the 1970s to more than $80 \%$ currently [1]. The increases have been achieved with more intensive and often toxic treatments, leading to concerns about the health-related quality of life (HRQL) of patients. Information about HRQL of patients should inform the development of therapeutic protocols with fewer or less severe disabilities. Although the literature is replete with reports about treatment effectiveness and the health of survivors, there are few reports describing the health of patients during phases of active treatment $[2,3]$ and none across all major discrete phases [4].

This study was undertaken to quantify the experiences of patients treated according to the Dana Farber Cancer Institute (DFCI) Childhood ALL Consortium 95-01 (DFCI 95-01) trial protocol [5], using the only generic utility-based instrument fulfilling a complete set of measurement performance criteria [6]: HEALTH UTILITIES INDEX® (HUI®) [Health Utilities Inc., 88 Sydenham Street, Dundas ON L9H-2V3, Canada]. Previous publications using HUI reported about patients with ALL during continuation (maintenance) therapy [7,8] or after the completion of treatment [9-16]. HUI health status was assessed from three perspectives. This report focuses on the parental perspective because many of the children were too young to self-report, the parental perspective is important in the clinical management of children's health, and there is ample evidence of acceptable reliability for parental assessments [14,16-20]. Quality-adjusted life years (QALYs) quantify the HRQLweighted time for each treatment phase.

At the onset of the study, the major hypotheses, informed by published evidence and clinical experience, were as follows. HRQL during active treatment would be low relative to the general population. HRQL would increase from a minimum during induction of remission to a maximum after completion of active treatment. Excess disability rates (rates of attributes at deficit levels for patients over-and-above control rates) for attributes of HRQL would be found in ambulation/mobility [7,16], emotion [7,10,11,13,14,16], cognition [9-11,13,14,16] and pain $[7,11,13,21]$. 


\section{METHODS}

\section{Study design}

The trial had a prospective cohort $(\mathrm{N}=491)$ design with a series of treatment randomizations. Therapy included four treatment phases (induction of 4 weeks duration, followed by CNS therapy of 3 weeks duration, intensification of 30 weeks duration and continuation of 71 weeks duration), administered in total for 25 months from diagnosis [5,22,23]. Standard and high risk patients were treated with one of two types of asparaginase, E.coli or Erwinia Lasparaginase, beginning in the induction phase. High risk patients received doxorubicin during induction with or without dexrazoxane, a potential cardioprotectant agent. Standard risk patients received treatments of the central nervous system (CNS) with or without cranial radiation and high risk patients received CNS treatment with hyper-fractionated or daily cranial radiation [5]. The HRQL study enrolled patients at diagnosis or, for patients too young to be eligible at diagnosis, at later assessments. HUI assessments were scheduled to provide representative measures of patients' health status for each phase.

\section{Subjects}

The study involved two types of subjects: ALL patients and general population control groups. ALL patients had to be 5 years of age or older at the time of assessment to be eligible for the HRQL study, as this is the minimum age recommended for use of HUI [8]. Five centers participated in the HRQL study (DFCI/Children's Hospital Boston, Boston MA, USA; Hôpital Sainte Justine, Montreal QC, Canada; Le Centre Hospitalier de L'Universite Laval, Quebec City QC, Canada; Maine Children's Cancer Program and Barbara Bush Children's Hospital at Maine Medical Center, Portland ME, USA; McMaster Children's Hospital, Hamilton ON, Canada). For control groups, we selected published summary results from a study country (Canada) with gender and age distributions similar to the sample of clinical participants: approximately equal numbers of males and females; ages 12 thru 16 years for HUI2 mean utility scores [24] and proportions of patients in disabled HUI2 attribute levels [25], ages 5 thru 12 years of age for HUI3 mean utility scores [21] and ages 15-19 for proportions of patients in disabled HUI3 attribute levels [26]. The research ethics review board for each participating institution approved the study and informed consent was obtained for each patient prior to enrolment.

\section{Measurement}

Clinical and demographic data were collected from medical records. Assessments were collected using standard self-complete HUI questionnaires with "past one-week" recall duration from three types of assessors according to published guidelines [27]: parent and clinician assessors for patients 5 years and older, as well as patient reports for those age 12 years and older. Assessors were eligible if literate in either English or Canadian French. Parental assessment perspective was defined for primary analyses by the original trial protocol to maximize the number of eligible patients, by including patients too young to self-report.

HUI assessments were collected for each of the following phases: remission induction (on day 23); CNS therapy (in the second week for those not requiring general anaesthesia or at 
initiation of the intensification phase); intensification (on week 3, day 14 of a cycle with asparaginase); continuation (on week 1, day 0 of a cycle); initial 2-years post-treatment (approximately 4 years after diagnosis); second 2-years post-treatment (approximately 6 years after diagnosis). HUI questionnaires collect information for both HUI Mark 2 (HUI2) and HUI Mark 3 (HUI3) health status classification systems [27]. Each classification system consists of a set of attributes, or domains, and a set of levels within each attribute. HUI2 has 7 attributes (sensation, mobility, emotion, cognition, self-care, pain, fertility) with each attribute having 3 to 5 levels [28]. HUI3 has 8 attributes (vision, hearing, speech, ambulation, dexterity, emotion, cognition, pain), each having 5 or 6 levels. The constructs of emotion, cognition and pain differ between HUI2 and HUI3. Emotion focuses on anxiety in HUI2 but depression in HUI3 [29,30], cognition on learning in HUI2 but solving day-to-day problems in HUI3, and pain on frequency in HUI2 and severity in HUI3. HUI2 fertility is not measured by HUI questionnaires and is considered uncompromised for calculating HRQL scores [27]. A comprehensive health state is a vector of one level for each attribute. Published utility functions were used to calculate two HRQL scores for each patient: one for the HUI2 comprehensive health state [31]; and one for the HUI3 comprehensive health state [32]. HRQL scores have interval-scale properties and represent mean community preferences on a scale such that dead $=0.00$, perfect health $=1.00$ [8]. QALYs, measuring combined quality and quantity of life, were calculated as the mean HRQL score times the treatment phase duration in years [33]. Disability categories for attribute levels are defined by validated schemes [34]. The clinically important difference between mean HRQL scores is $\geq 0.03[27,35]$.

\section{Data management and analysis}

HUI variables were derived from questionnaire responses according to standard algorithms [36]. Statistical analyses were completed using Minitab Release 13.20 and SPSS Release 15.0.1.

Treatment randomization groups were defined by intent-to-treat. Differences in means were assessed using analysis of variance. Chi-square and Fisher exact tests assessed differences of proportions. Statistical significance was set at the 5 percent level with Bonferroni adjustment for multiple testing. Mean HRQL scores provided quality-adjusted weights, and the trial protocols provided the durations, for calculating QALYs. Differences in QALYs between patient and control groups represent gains or losses of time-weighted HRQL for ALL patients. QALYs were transformed to quality-adjusted life days (QALDs), equivalent to days living in perfect health, to provide a readily interpretable metric. Attribute-specific excess disability rates (disability rates greater among patients than control groups), and differences in rates between phases, identify types and patterns of disability for remediation. Sampling effects were assessed by sensitivity analysis. HRQL score reliability was assessed by inter-rater agreement using the single-measure two-way intra-class correlation coefficient (ICC) and a published interpretive labeling scheme. ICC scores $<0.20$ represent poor agreement; 0.21 to 0.40 fair agreement; 0.41 to 0.60 moderate agreement; 0.61 to 0.80 good agreement; and 0.80 to 1.00 very good agreement [37]. 


\section{RESULTS}

\section{Subjects}

Demographic and clinical characteristics of the study population $(\mathrm{N}=375)$ are summarized in Table I. An accounting of eligibility, collected assessments and completed assessments is in Table II.

\section{Measurements}

The percent of overall trial patients eligible for HUI assessment varied across phases from a minimum of $46.9 \%$ during induction to a maximum of $84.3 \%$ during the initial 2-years posttreatment $(\mathrm{p}<0.001)$. Patient age less than 5 years was the primary reason for ineligibility during active treatment: $52.0 \%$ during induction; 51.2\% during CNS therapy; $49.9 \%$ during intensification; and 32.8\% during continuation. Disease relapse was the most common reason for exclusion during post-treatment phases: $10.4 \%$ during initial 2-years; and $12.8 \%$ during second 2-years.

Parental questionnaire completion rates varied among treatment phases from $71.6 \%$ during induction to $84.5 \%$ during continuation ( $\mathrm{p}=0.007$ ), and between post-treatment phases from $59.5 \%$ for initial 2-years to $38.6 \%$ for second 2-years ( $<<0.001$ ). The missing assessment rate varied from a minimum of $16 \%$ during continuation to a maximum of $62 \%$ for the second 2-years post-treatment. More than $99 \%$ of missing assessments were for unknown reasons. Because there were no significant differences in mean HRQL scores between the post-treatment phases ( $\mathrm{p}=0.651$ for HUI 2 and $\mathrm{p}=0.877$ for HUI3), and completion rates were less than $40 \%$ during the second 2-years, further analyses were limited to the treatment and initial 2-year post-treatment phases. There were no differences between the overall trial population ( $\mathrm{N}=491)$ and patients with HUI assessments (Table II) in the proportions of females and males during all phases ( $p>0.480$ ), and risk status during continuation and posttreatment phases ( $p>0.050)$. Standard risk patients with HRQL assessments were underrepresented relative to the trial population (55\%), due in large part to the exclusion of patients $<5$ years of age during: induction $(37 \%, \mathrm{p}<0.001)$; CNS therapy $(40 \%, \mathrm{p}=0.002)$; and intensification $(41 \%, \mathrm{p}=0.003)$. Patients were a mean age of $9.8($ minimum $=5.0$, maximum $=20.8$ ) years at assessment.

Mean HRQL increased from induction into the post-treatment phase (Table III). Differences in mean HRQL scores between ALL patients and the control groups were significant $(p<0.001)$ for each of the active treatment phases but not $(p>0.05)$ for the post-treatment phase. As is typical, mean HUI3 scores are lower than mean HUI2 scores [25].

Effects on HRQL scores of six patient factors, applicable during or before each phase, were tested to identify potential sub-groups for further analyses: gender; diagnostic risk group; age at diagnosis; type of asparaginase; CNS radiation fractions; cardioprotectant. There were no significant effects except during one phase for one factor: continuation, and the type of asparaginase received during intensification. The difference (E. coli minus Erwinia) in mean HRQL scores for HUI2 was $-0.05(0.88-0.93, \mathrm{p}=0.005)$ and for HUI3 was $-0.07(0.85-$ $0.92, \mathrm{p}=0.007)$. Thus, E. coli asparaginase was associated with more morbidity than the Erwinia product. Such differences were not apparent following completion of all treatment. 
However, it has been determined [5,38] that Erwinia asparaginase, when dosed once-weekly as it was in DFCI 95-01, was associated with a higher risk of relapse than E. coli; therefore, this dosing regimen for Erwinia is no longer a component of contemporary treatment protocols. For these reasons, subsequent analyses excluded continuation data of patients randomized to Erwinia and represent the experience of patients of contemporary interest: those receiving E. coli asparaginase.

QALYs for ALL survivors and control groups are illustrated in Figure 1. The upper panel shows HUI2 results and the lower panel HUI3 results. The upper bounds of the shaded areas represent the mean HRQL scores of controls and the lower bounds represent the mean HRQL scores of ALL patients. The shaded area represents the difference in QALYs experienced by controls and patients. The HUI2 results show that patients experience a loss, relative to controls, of 0.172 QALYs or 63 QALDs. Most (86\%) of the loss was during intensification (30\%) and continuation (56\%). The HUI3 results are similar but reveal a higher burden of morbidity.

The proportions of patients with varying numbers of disabled attributes differ across phases ( $p<0.001$ for each of HUI2 and HUI3): strongly skewed towards four or more disabled attributes during induction through strongly skewed towards zero disabled attributes during continuation. Disability rates of control and patient groups at induction (baseline) vary among attributes (Table IV). Excess disability rates of patients vary among attributes. Differences in excess rates vary among pairs of sequential phases. Excess disability rates decreased for HUI2 mobility, emotion (anxiety), self-care, and pain (frequency); and for HUI3 ambulation, emotion (depression), and pain (severity). Patterns differed for HUI2 emotion (anxiety) and HUI3 emotion (depression). There were residual excess disability rates during continuation for four HUI2 and four HUI3 attributes.

Sensitivity analyses of sampling effects compared the primary results based on all available HUI assessments to results based on the sub-group of patients having a complete set of HRQL assessments across treatment phases. During the four active treatment phases, there were 87 patients with a complete set of HUI2 scores and 86 patients with a complete set of HUI3 scores. The sub-sample was $43 \%$ female (similar to that of the study population), 9.8 years of age at diagnosis (tendency toward being older than the study population), and $63 \%$ high risk (tendency toward greater proportion of high risk than in the study population). The 95\% confidence intervals of all pairs of mean HRQL scores for the full sample and subsamples overlap, all the differences in mean HRQL scores were not clinically important (absolute difference $\$$ ).02), the directions of the differences in mean HRQL scores were not consistent across the assessment periods, and the difference in total QALY loss was $<7 \%$.

All ICCs of reliability indicated significant $(\mathrm{p}<0.05)$ inter-rater agreement. Mean ICCs across the 5 phases of parent-patient scores were $0.69(\min =0.54, \max =0.81)$ for HUI2 and $0.65(\min =0.49, \max =0.86)$ for HUI3; and of parent-clinician scores were $0.62(\mathrm{~min}=$ $0.51, \max =0.72)$ for HUI2 and $0.60(\min =0.49, \max =0.78)$ for HUI3. During all phases, parent-patient and parent-clinician agreement was moderate to very good. 


\section{DISCUSSION}

Patients experienced important deficits in HRQL during all treatment phases but not during the initial 2-years following treatment: severe deficits during induction of remission of disease, reflecting the combined effects of disease and treatment; moderate and decreasing deficits during CNS therapy through continuation treatment phases, reflecting remission of disease and declining treatment intensity; and mild deficits not significantly different from the general population after active treatment, reflecting no overall effects of active disease or treatment. HRQL deficits during active treatment were equivalent to losing approximately 2 months of life in perfect health, with $86 \%$ of the loss during intensification and continuation phases. The main explanatory attributes for increasing HRQL were mobility/ambulation, emotion, self-care, and pain.

The study demonstrates a trade-off of treatment effects: disease relapse rates; and acute health status. E. coli asparaginase treatment was associated with lower disease relapse rates [5] but greater morbidity relative to Erwinia asparaginase when both preparations are dosed once-weekly. Prevention of disease relapse has precedence over reduction in acute morbidity that is not severe and not potentially life-threatening. The greater burden of morbidity associated with E. coli, specifically in the attributes of HUI2 mobility and HUI3 ambulation during the intensification and continuation phases (Supplemental Table I), is consistent with the lower frequency of side effects with the Erwinia product when both are dosed once-weekly. It is possible that more frequent dosing of Erwinia asparaginase (known to have a shorter half-life than E. coli asparaginase) might be associated with efficacy and morbidity rates similar to that observed with once-weekly E. coli asparaginase. Why asparaginase-related morbidity differences persisted in the continuation phase of therapy, when no asparaginase was administered, is not clear, but may reflect persistent differential toxicity that is no longer evident after treatment with this drug has been completed.

There is one published parental assessment HUI-based study of continuation treatment [7]. The difference between mean HRQL of patients randomized to E. coli asparaginase in the current study (0.88) and those of DFCI 87-01 and 91-01 protocols (0.86) was not significant $(\mathrm{p}=0.320)$.

Four published studies reported mean HUI scores of HRQL for patients after completion of all treatment. There were no significant differences after adjusting for multiple testing between mean scores in the current study and each of three other studies [11-13], indicating that HRQL of survivors is similar across studies from Canada and Latin America. However, the mean score in the current study is 0.18 higher $(\mathrm{p}<0.001)$ than that of the study from Uruguay [16], indicating that the HRQL of ALL survivors is poorer in Uruguay than elsewhere.

Contrary to the results of the current study are three published reports of important differences in mean HUI scores of HRQL between ALL patients after completion of therapy and comparison/control groups. All three report ALL survivors having lower HRQL than controls. Two reports, from one underlying study of Canadian patients, reported differences of $0.05(\mathrm{p}<0.001)$ for HUI2 and $0.10(\mathrm{p}<0.001)$ for HUI3, with point estimates for mean 
control scores being similar $(\mathrm{p}=0.340)$ and ALL patient scores being lower $(\mathrm{p}=0.036)$ than in the current study $[12,13]$. The other study, from Uruguay [16], reported a controls minus ALL survivors difference in means of $0.08(\mathrm{p}=0.018)$ for HUI3, with mean scores for both controls and ALL survivors being $\searrow 0.15$ lower than in the current study $(\mathrm{p}<0.001)$. Differences in mean HRQL between controls and ALL survivors across the 3 studies are somewhat inconsistent in size and not entirely unexpected given the variability in many study design factors including country, assessment perspective and language, and mode of questionnaire administration. The previous studies used convenience samples for controls and had relatively small sample sizes, compared to the current study, but the most important factor may be time off therapy for patients. Time off therapy in the current study was relatively short, 2.0 years, compared with 1.0 to 13.6 (mean $=5.4$ ) years for the Canadian patients and 4.9 to 7.5 years for the Uruguayan patients. Two years off-therapy may be too short for disabilities to be manifested and detected.

Important excess disability rates in ambulation/mobility, emotion and pain during active treatment were reported previously among patients during continuation phases of DFCI 87-01 and 91-01 protocols [7]. Excess disability rates in cognition, self-care, speech and dexterity during active treatment phases represent new reports.

Important strengths of the current study include the use of two well-validated health measurement systems with demonstrated intra-study reliability, a large multi-center study population with contemporary treatment, large samples during active and off-treatment phases, assessments from each discrete treatment phase, acceptable survey rates, and commensurate results for control groups. Patients assessed were representative of the trial population for gender and risk group status during the majority of treatment duration.

Limitations of this study include no HRQL measurements for patients $<5$ years of age, after disease relapse, after bone marrow transplantation, and after initiation of palliative care. The data suggest (Supplemental Table II) that the HRQL of patients with standard risk disease may be greater than that of patients with high risk disease during and after the intensification phase (high risk treatment is more intensive than standard risk treatment only during the intensification phase) but this study may lack the power to detect the difference after adjusting for multiple testing. This study did not detect a difference in overall HRQL between ALL survivors during the post-treatment phase and control groups, but this should not be interpreted as evidence that the health of the survivors will remain similar to the general population because adult survivors of cancer in childhood have high rates of chronic conditions, some of which develop many years after the completion of treatment [39].

Controls were not matched to patients at the individual level because recruiting controls was beyond the scope of the trial that focused on assessing the HRQL experience of patients. Finally, response rates for some phases were less than ideal, so generalizability of the results may be limited.

This study reports on the comprehensive health status and HRQL assessments of patients during all major phases of treatment for ALL in childhood, providing a much more complete picture than was previously available in the literature. Children with ALL experience very large HRQL deficits over the course of treatment: equivalent to losing approximately 2 
months of life in perfect health. Most of this loss is during the intensification and continuation phases. Multiple disabilities are common. High excess disability rates for ambulation/mobility, emotion and pain indicate there is much scope for improvement in these areas. This study did not detect important HRQL deficits among survivors during the 2-year post-treatment phase, but this should not be extrapolated to longer term survivors because other studies report higher rates of chronic conditions and/or health problems among survivors.

The policy challenge is to focus on the development of treatment regimens involving fewer and less severe disabilities, especially during intensification and continuation phases, without negatively impacting rates of relapse and overall survival. Current treatment protocols for ALL in children and adolescents contain many components that are similar to DFCI 95-01, so the conclusions from this study are relevant to contemporary therapies.

\section{Supplementary Material}

Refer to Web version on PubMed Central for supplementary material.

\section{Acknowledgments}

Grants from the National Institutes of Health (CA68484 and CA006516) and Garil Fund. Recruitment by Drs. Craig Hurwitz, Albert Moghrabi and Yvan Samson. Questionnaire completion by patients, parents and clinicians.

\section{REFERENCES}

1. National Cancer Policy Board (USA). Childhood cancer survivorship: improving care and quality of life. Hewitt, M.; Weiner, SL.; Simone, JV., editors. The National Academies Press; Washington, DC: 2003. ISBN 0309088984

2. Barr RD, Furlong W, Feeny D, et al. Evaluating treatments for childhood cancer: a process for critical appraisal of the literature and a summary of the results. Int J Technol Assess Health Care. 1995; 11:1-10. [PubMed: 7706006]

3. Pickard AS, Topfer L-A, Feeny DH. A structured review of studies on health-related quality of life and economic evaluation in pediatric acute lymphoblastic leukemia. J Nat Cancer Inst Monogr. 2004; 33:102-125.

4. Sung L, Yanofsky R, Klaassen RJ, et al. Quality of life during active treatment for pediatric acute lymphoblastic leukemia. Int J Cancer. 2011; 128:1213-1220. [PubMed: 20473921]

5. Moghrabi A, Levy DE, Asselin B, et al. Results of the Dana-Farber Cancer Institute ALL Consortium Protocol 95-01 for children with acute lymphoblastic leukemia. Blood. 2007; 109:896904. [PubMed: 17003366]

6. Eiser C, Morse R. The measurement of quality of life in children: past and future perspectives. J Dev Behav Pediatr. 2001; 22:248-256. [PubMed: 11530898]

7. Barr RD, Petrie C, Furlong W, et al. Health-related quality of life during post-induction chemotherapy in children with acute lymphoblastic leukemia in remission: an influence of corticosteroid therapy. Int J Oncol. 1997; 11:333-339. [PubMed: 21528219]

8. Furlong WJ, Feeny DH, Torrance GW, et al. The Health Utilities Index $\left(\mathrm{HUI}^{\circledR}{ }^{\circledR}\right.$ ) system for assessing health-related quality of life in clinical studies. Ann Med. 2001; 33:375-384. [PubMed: 11491197]

9. Barr RD, Furlong W, Dawson S, et al. An assessment of global health status in survivors of acute lymphoblastic leukemia in childhood. Am J Pediatr Hematol Oncol. 1993; 15:284-290. [PubMed: 8328641] 
10. Feeny DH, Leiper A, Barr RD, et al. The comprehensive assessment of health status in survivors of childhood cancer: application to high-risk acute lymphoblastic leukaemia. Br J Cancer. 1993; 67:1047-1052. [PubMed: 8494697]

11. Barr RD, Gonzalez A, Longchong M, et al. Health status and health-related quality of life in survivors of cancer in childhood in Latin America: a MISPHO feasibility study. Int J Oncol. 2001; 19:413-421. [PubMed: 11445861]

12. Wright MJ, Galea V, Barr RD. Self-perceptions of physical activity in survivors of acute lymphoblastic leukemia in childhood. Pediatr Exerc Sci. 2003; 15:191-201.

13. Wright MJ, Galea V, Barr RD. Proficiency of balance in children and youth who have had acute lymphoblastic leukemia. Phys Ther. 2005; 85:782-790. [PubMed: 16048425]

14. Fu L, Talsma D, Baez F, et al. Measurement of health-related quality of life in survivors of cancer in childhood in Central America: feasibility, reliability, and validity. J Pediatr Hematol Oncol. 2006; 28:331-341. [PubMed: 16794499]

15. Shimoda S, Horsman J, Furlong W, et al. Disability and health-related quality of life in long-term survivors of cancer in childhood in Brazil. J Pediatr Hematol Oncol. 2008; 30:563-570. [PubMed: 18799931]

16. Fluchel M, Horsman JR, Furlong W, et al. Self and proxy-reported health status and health-related quality of life in survivors of childhood cancer in Uruguay. Pediatr Blood Cancer. 2008; 50:838843. [PubMed: 17635006]

17. Barr RD, Simpson T, Whitton A, et al. Health-related quality of life in survivors of tumours of the central nervous system in childhood - a preference-based approach to measurement in a crosssectional study. Eur J Cancer. 1999; 35:248-255. [PubMed: 10448267]

18. Glaser AW, Furlong W, Walker DA, et al. Applicability of the Health Utilities Index to a population of childhood cancer survivors of central nervous system tumours in the United Kingdom. Eur J Cancer. 1999; 35:256-261. [PubMed: 10448268]

19. Verrips GHW, Stuifbergen MC, den Ouden AL, et al. Measuring health status using the Health Utilities Index: agreement between raters and between modalities of administration. J Clin Epidemiol. 2001; 54:475-481. [PubMed: 11337210]

20. Banks BA, Barrowman NJ, Klaassen R. Health-related quality of life in children undergoing chemotherapy. J Pediatr Hematol Oncol. 2008; 30:292-297. [PubMed: 18391698]

21. Pogany L, Barr RD, Shaw A, et al. Health status in survivors of cancer in childhood and adolescence. Qual Life Res. 2006; 15:143-157. [PubMed: 16411038]

22. U.S. National Institutes of Health. Registry of clinical trials: Treatment of childhood acute lymphoblastic leukemia, 12/07 update. http://clinicaltrials.gov/ct2/show/NCT00165087

23. Waber DP, Turek J, Catania L, et al. Neuropsychological outcomes from a randomized trial of triple intrathecal chemotherapy compared with 18 Gy cranial radiation as CNS treatment in acute lymphoblastic leukemia: findings from Dana-Farber Cancer Institute ALL Consortium Protocol 95-01. J Clin Oncol. 2007; 25:4914-4921. [PubMed: 17971588]

24. Feeny D, Furlong W, Saigal S, et al. Comparing directly measured standard gamble scores to HUI2 and HUI3 utility scores: group and individual-level comparisons. Soc Sci Med. 2004; 58:799-809. [PubMed: 14672594]

25. Saigal S, Rosenbaum PL, Feeny D, et al. Parental perspectives of the health status and healthrelated quality of life of teen-aged children who were extremely low birth weight and term controls. Pediatrics. 2000; 105:569-574. [PubMed: 10699111]

26. Statistics Canada. Health Status of Canadians: Report of the 1991 General Social Survey. Ottawa, Canada, Statistics Canada: 1994. Housing, Family and Social Statistics Division. General Social Survey Analysis Series.. Cat. 11-612E, No. 8

27. Horsman J, Furlong W, Feeny D, et al. The Health Utilities Index (HUI®): concepts, measurement properties and applications. Health Qual Life Outcomes. 2003; 1:54. http://www.hqlo.com/ content/1/1/54. [PubMed: 14613568]

28. Feeny D, Furlong W, Barr RD, et al. A comprehensive multiattribute system for classifying the health status of survivors of childhood cancer. J Clin Oncol. 1992; 10:923-928. [PubMed: 1316952] 
29. Revicki DA, Brandenburg N, Matza L, et al. Health-related quality of life and utilities in primarycare patients with generalized anxiety disorder. Qual Life Res. 2008; 17:1285-1295. [PubMed: 18949580]

30. Feeny D, Huguet N, McFarland B, et al. The construct validity of the Health Utilities Index Mark 3 in assessing mental health in population health surveys. Qual Life Res. 2009; 18:519-526. [PubMed: 19277898]

31. Torrance GW, Feeny DH, Furlong WJ, et al. Multi-attribute preference functions for a comprehensive health status classification system: Health Utilities Index Mark 2. Med Care. 1996; 34:702-722. [PubMed: 8676608]

32. Feeny D, Furlong W, Torrance GW, et al. Multi-attribute and single-attribute utility functions for the Health Utilities Index Mark 3 system. Med Care. 2002; 40:113-128. [PubMed: 11802084]

33. Torrance GW, Feeny D. Utilities and quality-adjusted life years. Int J Technol Assess Health Care. 1989; 5:559-575. [PubMed: 2634630]

34. Feng Y, Bernier J, McIntosh C, et al. Validation of disability categories derived from Health Utilities Index Mark 3 scores. Health Rep. 2009; 20:43-50. (Statistics Canada, Catalogue 82-003XPE). [PubMed: 19728585]

35. Revicki DA, Feeny D, Hunt TL, et al. Analyzing oncology clinical trials data using the Q-TWiST method: clinical importance and sources for health state preference data. Qual Life Res. 2006; 15:411-423. [PubMed: 16547779]

36. Furlong, WJ.; Feeny, DH.; Torrance, GW. HEALTH UTILITIES INDEX ${ }^{\circledR}$ (HUI®) Procedures Manual: Algorithm for determining HUI Mark 2 (HUI2) / Mark 3 (HUI3) health status classification levels, health states, single-attribute level utility scores and overall health-related quality of life utility scores from 15-item self-complete HUI health status questionnaires.. Health Utilities Inc.; Dundas, ON, Canada: 2000.

37. Altman, DG. Practical Statistics for Medical Research. Chapman \& Hall; London: 1991.

38. Duval M, Suciu S, Ferster A, et al. Comparison of Escherichia coli-asparaginase with Erwiniaasparaginase in the treatment of childhood lymphoid malignancies: results of a randomized European Organization for Research and Treatment of Cancer - Children's Leukemia Group phase 3 trial. Blood. 2002; 99:2734-2739. [PubMed: 11929760]

39. Oeffinger KC, Mertens AC, Sklar CA, et al. Chronic health conditions in adult survivors of childhood cancer. N Engl J Med. 2006; 355:1572-1582. [PubMed: 17035650] 
HU12

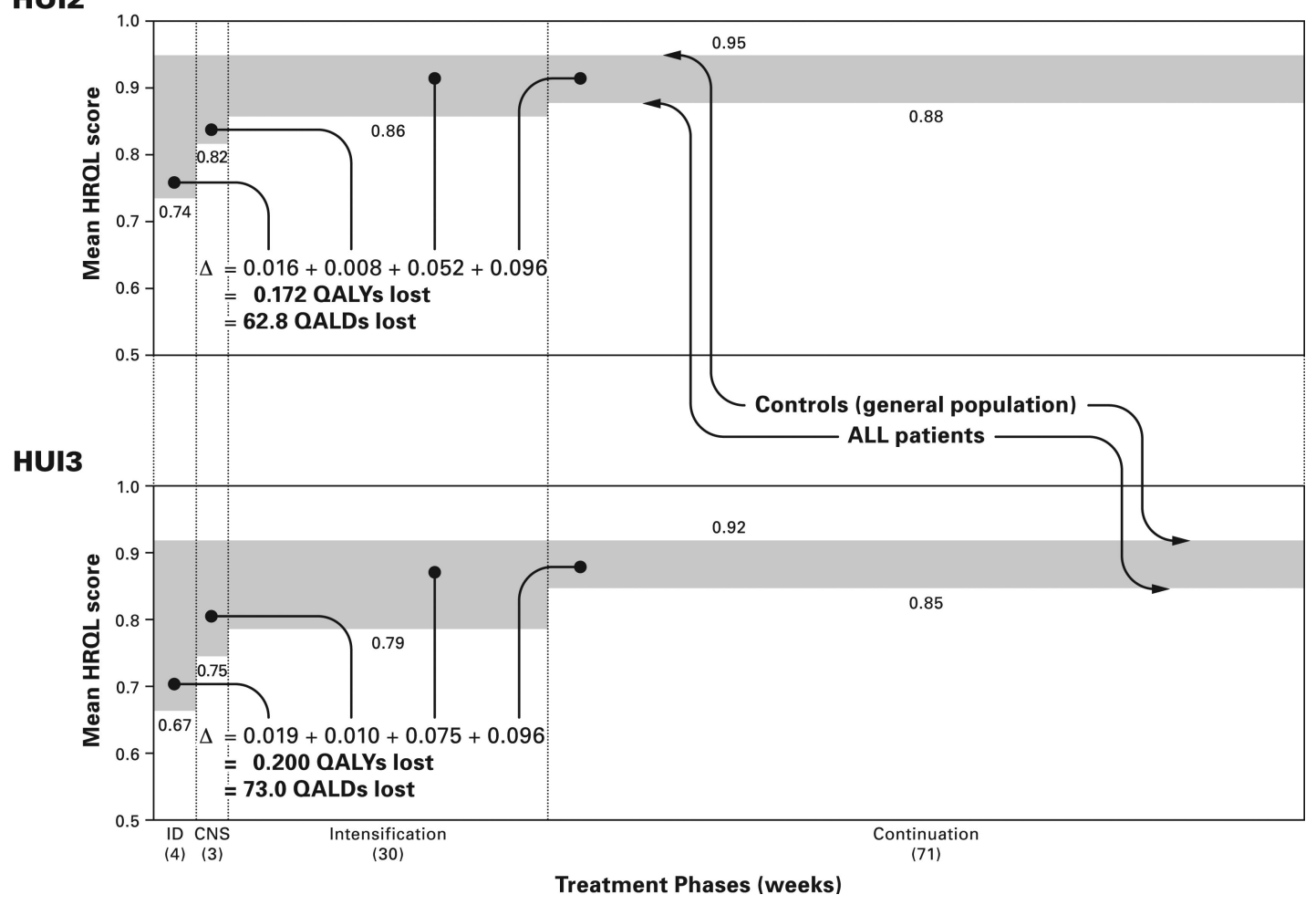

Fig. 1.

Quality-adjusted life years (QALYs) and days (QALDs) lost by ALL patients during active treatment phases according to HUI2 and HUI3 systems. During induction, CNS and intensification treatment phases there were no differences in mean HRQL scores between E. coli and Erwinia asparaginase treatment groups so the results for these 3 phases are based on pooled data from both groups. During continuation there were significant differences in mean HRQL between the asparaginase groups so the results for this phase are based on data from only the E.coli group. 
TABLE I

Demographic and Clinical Characteristics of the Patient Population (N=375)

\begin{tabular}{|c|c|c|c|}
\hline \multirow{2}{*}{\multicolumn{2}{|c|}{$\frac{\text { Characteristic }}{\text { Age at Diagnosis (years) }}$}} & \multicolumn{2}{|c|}{$\underline{\text { Statistics }}$} \\
\hline & & Mean & 6.11 \\
\hline & Median & 4.64 \\
\hline & & Standard deviation & 4.25 \\
\hline & & Minimum & 0.04 \\
\hline & & Maximum & 18.00 \\
\hline & & \multicolumn{2}{|c|}{ Frequency Distributions } \\
\hline$\underline{\text { Characteristic }}$ & $\underline{\text { Category }}$ & $\underline{\mathbf{n}}$ & $\underline{\%}$ \\
\hline \multirow[t]{2}{*}{ Gender } & Females & 168 & 44.8 \\
\hline & Males & 207 & 55.2 \\
\hline \multirow[t]{5}{*}{ Treatment Center * } & DFCI & 135 & 36.0 \\
\hline & Sainte Justine & 131 & 34.9 \\
\hline & Laval & 36 & 9.6 \\
\hline & Maine & 28 & 7.5 \\
\hline & McMaster & 45 & 12.0 \\
\hline \multirow[t]{2}{*}{ Diagnostic Group } & Standard risk & 199 & 53.1 \\
\hline & High risk & 176 & 46.9 \\
\hline \multirow[t]{3}{*}{ Treatment Response } & Complete remission & 364 & 97.0 \\
\hline & Induction failure & 7 & 1.9 \\
\hline & Induction death & 4 & 1.1 \\
\hline \multirow[t]{3}{*}{ Relapse } & No & 321 & 85.6 \\
\hline & Yes & 48 & 12.8 \\
\hline & Unknown & 6 & 1.6 \\
\hline \multirow[t]{3}{*}{ Status at End Of Study } & Alive & 340 & 90.7 \\
\hline & Deceased & 32 & 8.5 \\
\hline & Unknown / Lost to follow-up & 3 & 0.8 \\
\hline
\end{tabular}

$\mathrm{n}$, number of patients;

*

*, full names of treatment centers are in methods section of text. 


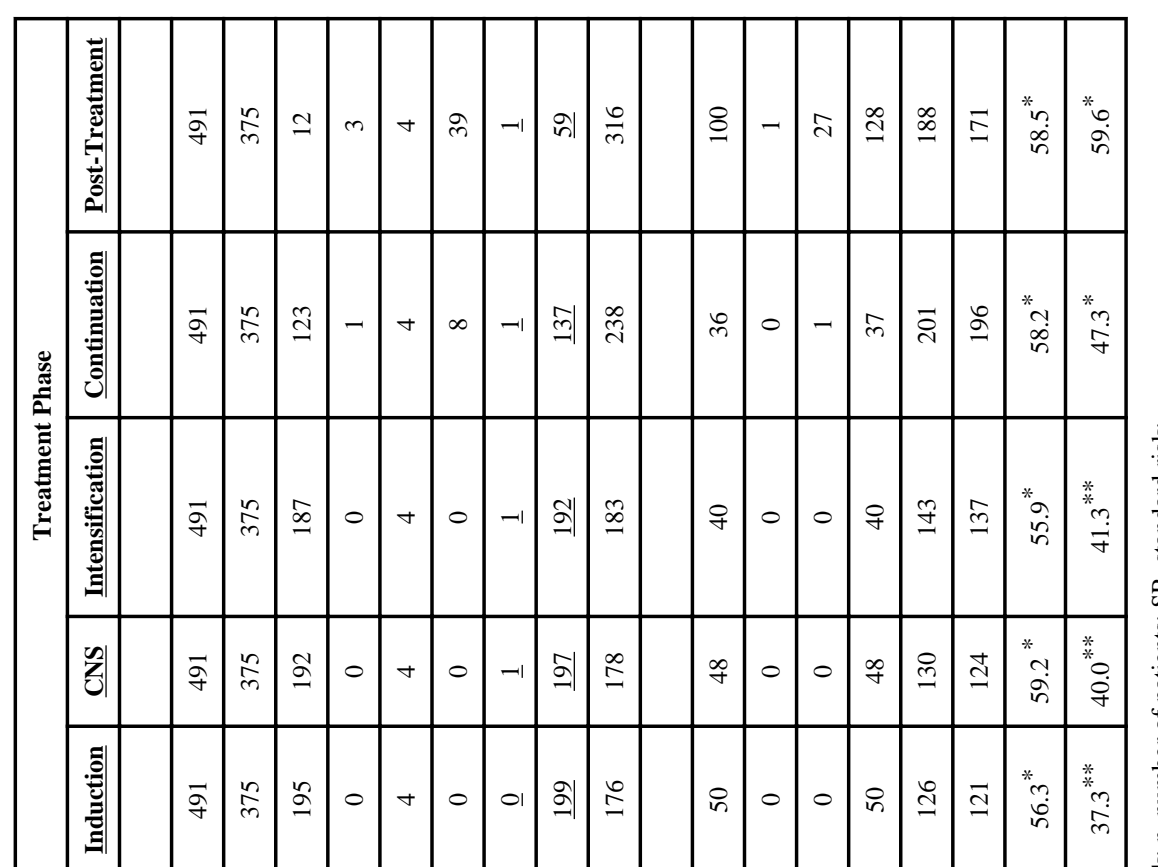

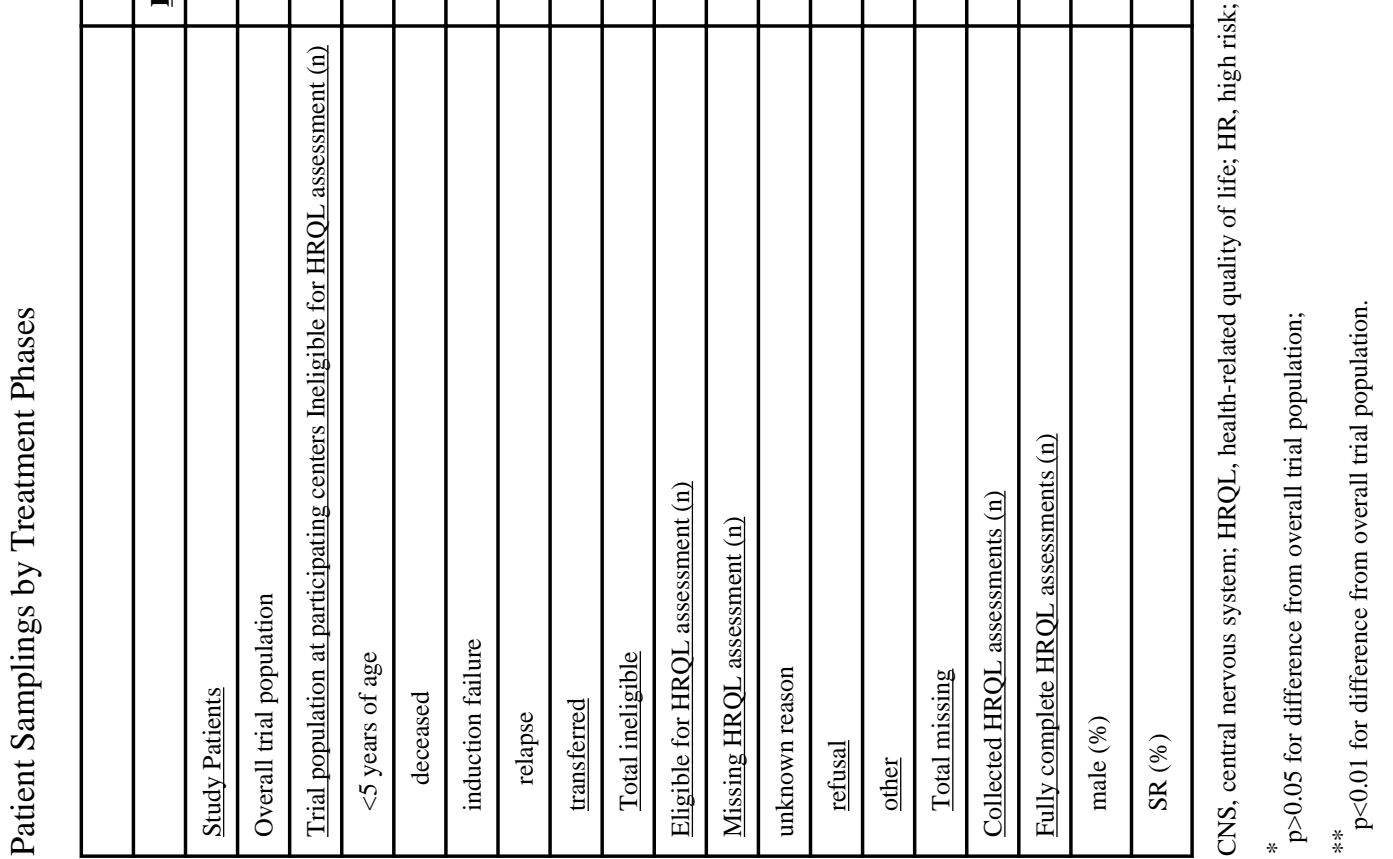




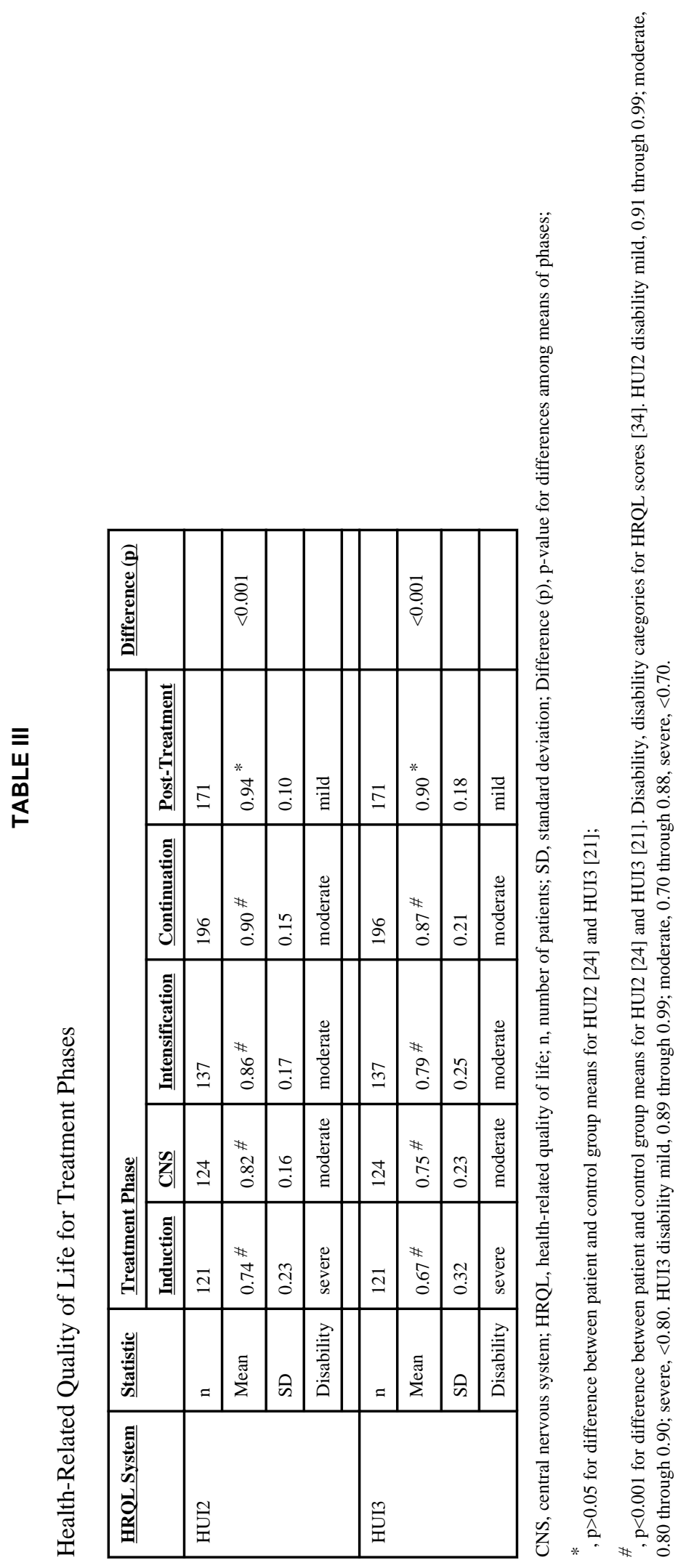

Pediatr Blood Cancer. Author manuscript; available in PMC 2014 August 06. 


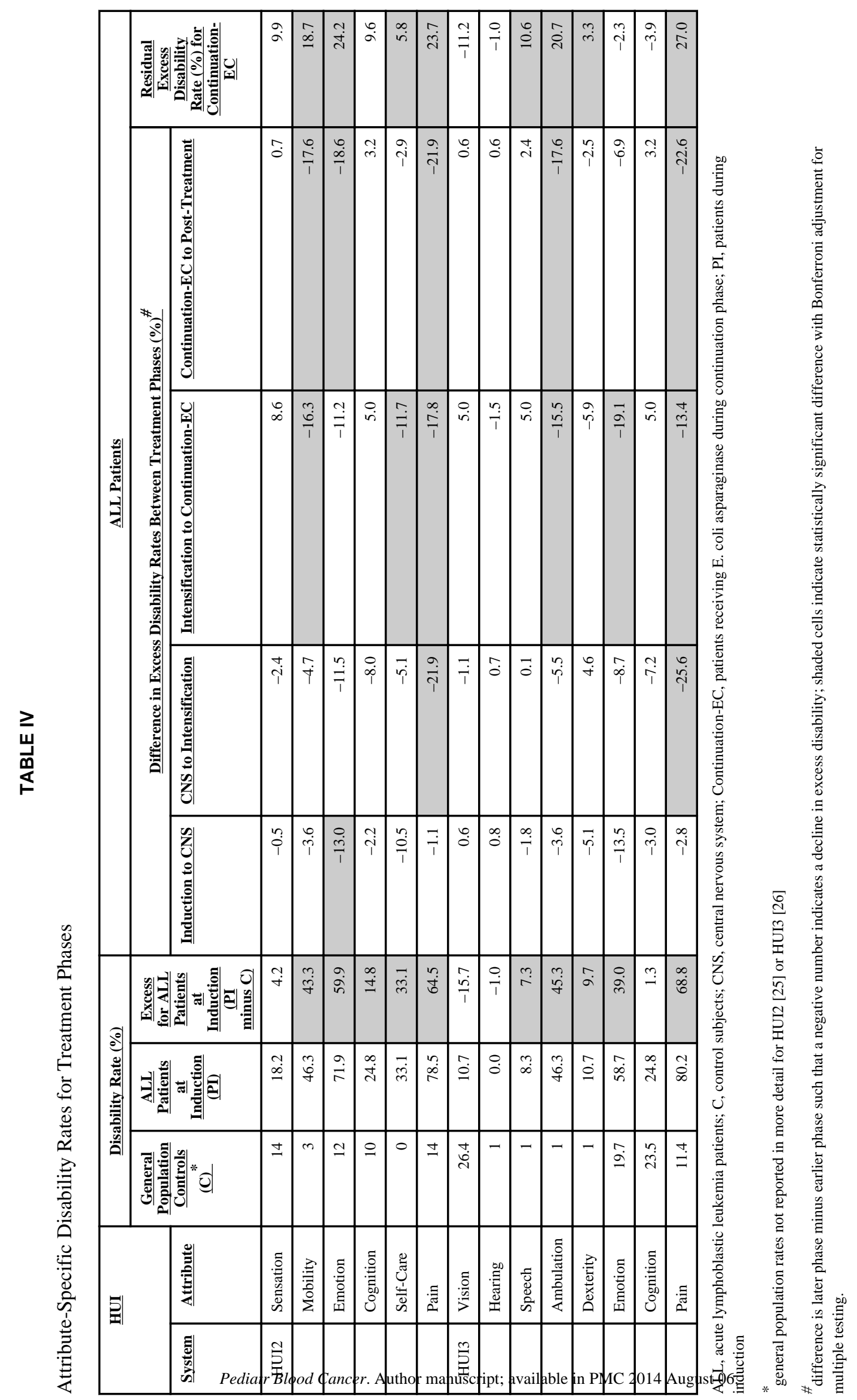

